\title{
Efficacy of a twice-daily, 3-step, over-the-counter skincare regimen for the treatment of acne vulgaris
}

\author{
Katie Rodan \\ Kathy Fields \\ Timothy J Falla \\ Rodan + Fields, San Francisco, \\ CA, USA
}

This article was published in the following Dove Press journal:

Clinical, Cosmetic and Investigational Dermatology

4 January 2017

Number of times this article has been viewed
Background: Acne vulgaris (acne) is the most common skin disorder producing physical and emotional scars that can persist for years. An estimated $83 \%$ of acne sufferers self-treat, but there is lack of studies documenting the effectiveness of over-the-counter (OTC) acne treatment products. Objective: This study was conducted to determine the effectiveness of an OTC, 3-step, antiacne skincare regimen in treating acne and improving the appearance of red/inflamed facial skin. Methods: This 6-week, open-label clinical study included both genders aged between 12 and 35 years with mild-to-moderate acne. All subjects were required to have an acne score of 1-3 (Cook's acne grading scale: $0=$ clear to $7=$ very severe) and a moderate redness score of $\geq 2$ $(0=$ none and $4=$ severe $)$. Subjects completed a 3-step facial treatment regimen every morning and evening using an OTC cleanser, toner, and acne treatment. Evaluations for effectiveness and safety were done at baseline and weeks 2, 4, and 6 using digital photographs (Visia-CR ${ }^{\circledR}$ digital imaging system) of the face and analyzed using Image-Pro ${ }^{\circledR}$ software for the grading of acne, red/inflamed skin, and the number and type of lesions.

Results: Thirty subjects (12 males and 18 females) were enrolled (mean age of 19 years; range 12-34 years). This skincare regimen resulted in statistically significant improvements in acne grading scores after 2 weeks of use, with mean scores continuing to improve after 4 and 6 weeks of use $(P<0.001)$. Statistically significant improvements from baseline in red/inflamed skin, open and closed comedones, and papules were detected at all time points and for nodules at week 6, compared to their respective baselines $(P<0.05)$.

Conclusion: This clinical study demonstrated the effectiveness of an OTC 3-step, anti-acne skincare regimen in significantly improving acne and the overall appearance of skin in the majority of subjects who had mild-to-moderate acne.

Keywords: acne, acne treatment, skincare regimen, over-the-counter

\section{Introduction}

Acne vulgaris (acne) is the most common skin disorder in the USA, affecting up to 50 million people and $\sim 85 \%$ of people aged $11-30$ years. ${ }^{1-3}$ Acne is characterized by noninflammatory (open or closed comedones) and inflammatory lesions (papules, pustules, or nodules). ${ }^{3}$ Grading, extent, and morphology of these lesions determine acne severity and help guide therapy choices. ${ }^{3}$

Although acne affects people of all ages, it is most prevalent during adolescence. Because facial acne is difficult to camouflage, it can produce lasting physical and emotional scars, which impact self-image, create a barrier to interpersonal relationships, and promote social biases. ${ }^{1,4,5}$ Those suffering from acne tend to have feelings of isolation and loneliness with an increased risk for anxiety and depression. ${ }^{1,4,5}$
Correspondence: Timothy J Falla Rodan + Fields, 60 Spear Street, San Francisco, CA 94105, USA

Tel + I 4I5 5976328

Email tfalla@rodanandfields.com 
Studies have further demonstrated a reduced quality of life, similar to people with asthma, epilepsy, diabetes, back pain, or arthritis. ${ }^{6}$ Interestingly, detrimental psychological effects do not correlate with acne severity; even mild forms of acne can have significant psychosocial impacts. ${ }^{1}$

Treating acne at its earliest stages reduces overall morbidity. ${ }^{1}$ Treatment goals include a decrease in the severity and recurrence rate of noninflammatory and inflammatory lesions, prevention of permanent scars, improvement of overall appearance, and reduction of associated psychological impact., ${ }^{3,7}$

In a study of adolescent acne, $83 \%$ of sufferers never saw a physician for treatment, instead opting to self-treat. ${ }^{8}$ There are many over-the-counter (OTC) acne treatments available designed to target at least one of the four factors that cause the disease (increased sebum production, follicular hyperkeratinization, bacterial colonization, and inflammation). ${ }^{3,9}$ When properly selected, OTC treatments have the advantage of being convenient, inexpensive, and effective for mild-tomoderate disease. ${ }^{9}$

Because OTC products are not tested with the same rigor as prescription drugs, data regarding the effectiveness of OTC acne treatment options are limited. ${ }^{3,10}$ As a result, dermatologists and other health care providers often have difficulty differentiating and recommending the most appropriate products for their patients. $^{9}$

This study was conducted to determine the effectiveness of an OTC 3-step anti-acne skincare regimen in treating acne and improving the appearance of red/inflamed facial skin.

\section{Methods}

\section{Study population}

Males and females aged between 12 and 35 years with mild-to-moderate acne were recruited to participate in this single-center, 6-week, open-label clinical study.

An independent, board-certified dermatologist evaluated the facial area of all subjects for acne, redness, and lesions to determine study eligibility. All study participants were required to have an acne score of 1-3 according to Cook's acne grading scale $(0=$ clear and $7=$ very severe $)$ and a moderate redness score of $\geq 2(0=$ none and $4=$ severe $)$ but could not have had any other dermatological conditions that could have interfered with the study. ${ }^{11}$

Subjects were ineligible if, prior to the start of the study, they had used an OTC acne treatment within the previous 4 weeks, prescription acne treatment (eg, isotretinoin, tretinoin, and antibiotics) within the previous 6 months, or any systemic or topical anti-inflammatory agents within the previous 5 days (with the exception of acetaminophen, ibuprofen, or aspirin). Subjects were instructed not to use any acne treatments other than those supplied for use during the 6-week study.

This study was designed to assess an anti-acne skincare regimen in people of diverse skin types and ethnicities (Caucasian, African American, Asian, and Latin American ethnicity). Eligibility criteria were structured to ensure at least $60 \%$ of the subjects were Caucasian. Eligible subjects were required to be in general good health with no known sensitivities to cosmetics or personal care products. They were ineligible if they had any systemic or dermatological disorders, including a history of allergies, that could interfere with the conduct of the study and interpretation of the results, or increase the risk of adverse reactions. Female subjects were ineligible if they were pregnant, planning to become pregnant during the study, or lactating/nursing.

\section{Study design}

All subjects who met the eligibility criteria were instructed to use an OTC 3-step facial treatment regimen every morning and evening. The regimen consisted of a cleanser (Step 1), a toner (Step 2), and acne treatment (Step 3). Specific instructions for how to use the 3-step regimen, given to each subject, are provided in Supplementary material S1. Optional moisturizer was provided for use after Step 3, if dryness or redness occurred. Sunscreen was also offered as an option for use after Step 3, with or without moisturizer use.

The key ingredients of the treatment materials were as follows: cleanser: sulfur 3\%; toner: witch hazel water, ethoxydiglycol, aloe lead juice, glycolic acid, sodium hydroxide, and sodium hyaluronate; and acne treatment: two separate formulas, cream (benzoyl peroxide 5\%) and gel containing ethoxydiglycol, dispensed individually in equal quantities (1:1 ratio) from two sides of the packaging.

All subjects were evaluated at baseline and weeks 2, 4, and 6 for effectiveness and safety of the test materials. At all study visits, digital photographs of the face were analyzed using the Visia-CR ${ }^{\circledR}$ digital imaging system (Canfield Scientific, Parsippany, NJ, USA). ${ }^{12,13}$ To ensure consistency, each subject was draped with a black cloth around the shoulders and wore a black headband to pull hair away from the face. All subjects were free of any makeup during evaluations, and images were taken of the front, right, and left views. The images were analyzed using Image-Pro ${ }^{\circledR}$ software (Media Cybernetics, Rockville, MD, USA) for acne grading, red/ inflamed skin, and the number and type of lesions.

Acne scores were graded according to Cook's acne grading scale. Differences in between-visit scores were calculated, 
with a decrease in score representing acne improvement and an increase representing worsening. Skin redness/inflammation was determined by the Visia-CR ${ }^{\circledR}$ system, in which the Commission Internationale de l'Eclairage (CIE) $a^{*}$ value corresponded to skin redness/erythema. ${ }^{14}$ The differences in CIE a* values between visits were calculated, with a decrease in value indicating an improvement (ie, whitening or reduced red effect) and an increase indicating a worsening (ie, darkening or increased red effect). The number and type of adverse conditions were documented from investigator observations and the subjects' self-reporting and diary comments.

This study was conducted by Essex Testing Inc, Verona, NJ, USA, in compliance with the International Conference on Harmonization Good Clinical Practice guidelines and the ethical principles of the Declaration of Helsinki. Before the start of the study, all subjects gave their written informed consent in compliance with all applicable local and federal regulations. Parents or legal guardians gave written informed consent on behalf of subjects who were younger than 18 years.

\section{Statistical analysis}

All subjects who received treatment materials and completed the study in accordance with the protocol (per-protocol population) were included in the statistical analyses. A study duration of 6 weeks was selected based on the experience of the authors in which response to treatment is usually observed within 6 weeks or less. Descriptive statistics (ie, mean, standard deviation [SD], and range) were used to evaluate the acne grading scores, red/inflamed skin scores, and lesion count measurements. For each measure, mean change

$$
\text { A }
$$

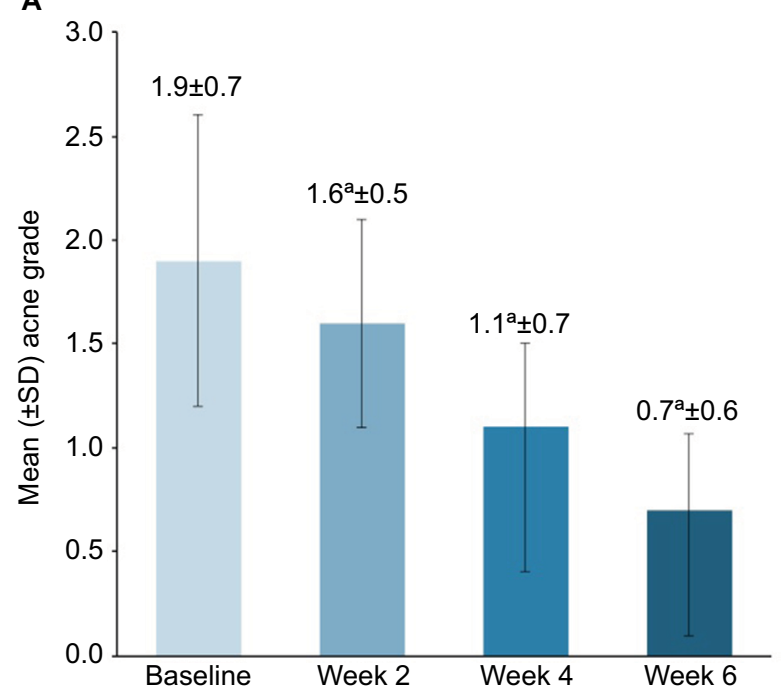

from baseline, percent age mean change from baseline, and percentage of subjects showing improvement or worsening were calculated for each postbaseline time point. The null hypothesis that the mean change from baseline is zero was tested using a Wilcoxon signed-rank test and paired $t$-tests, with a statistical significance level $\alpha=0.05$.

\section{Results \\ Study population}

A total of 30 subjects, 12 males and 18 females, met the eligibility criteria and were enrolled, with all subjects completing the study. Mean age was 19 years (range 12-34 years), and the ethnicity distribution was White, $n=21$ (70\%); Hispanic, $n=5(17 \%)$; and other, $n=4(13 \%)$.

Eligibility baseline parameters as assessed by dermatologists, per the study design, were as follows: all subjects had a Cook's acne grading scale score of $1-3$, with 15 (50\%), 13 $(43 \%)$, and $2(7 \%)$ having scores of 1,2 , and 3 , respectively; mean skin redness was 2.2 (range 2-3), indicating moderateto-marked facial redness; and mean lesion counts were as follows: open comedones 8.1 , closed comedones 1.1 , papules 2.3 , pustules 0.3 , and nodules 0.1 , for a mean total lesion count of 11.9 (range 7-18).

Digital photographs using the Visia-CR ${ }^{\circledR}$ imaging system and analyzed by the Image-Pro ${ }^{\circledR}$ software detected statistically significant improvements in acne grading scores at 2 weeks, with mean scores continuing to improve after 4 and 6 weeks of use $(P<0.001$ for all time points; Figure 1$)$. Baseline mean score $( \pm \mathrm{SD})$ was $1.9 \pm 0.7$, which improved (decreased) to $1.6 \pm 0.5,1.1 \pm 0.7$, and $0.7 \pm 0.6$ at weeks 2 ,

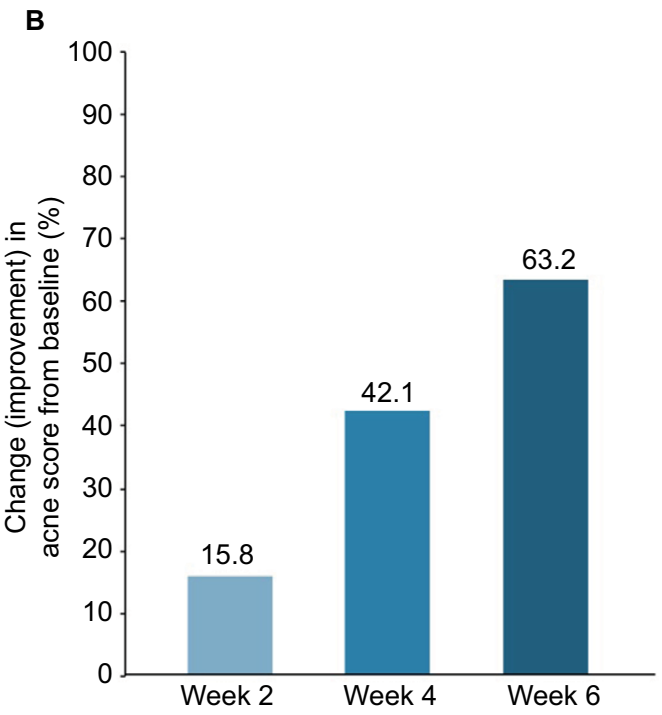

Figure I Improvement in (A) mean acne grade scores and (B) percentage change from baseline of acne grade scores in subjects who used twice-daily, 3-step anti-acne skincare regimen.

Notes: ${ }^{a} P<0.05$ versus baseline. Visia-CR ${ }^{\circledR} /$ Image-Pro ${ }^{\circledR}$ analysis.

Abbreviation: SD, standard deviation. 
4, and 6, respectively (Figure 1). This resulted in a mean percentage change (improvement) from baseline of $15.8 \%$ at week 2 , increasing to $42.1 \%$ and $63.2 \%$ at weeks 4 and 6 , respectively (Figure 1).

One-third $(n=10 ; 33 \%)$ of subjects experienced acne improvement after 2 weeks of treatment. With continued treatment, $70 \%(n=21)$ of subjects experienced acne improvement after 4 weeks, and almost all subjects $(n=27 ; 90 \%)$ experienced improvement after 6 weeks.

Statistically significant improvements from baseline in red/ inflamed skin were detected at all time points after subjects

A

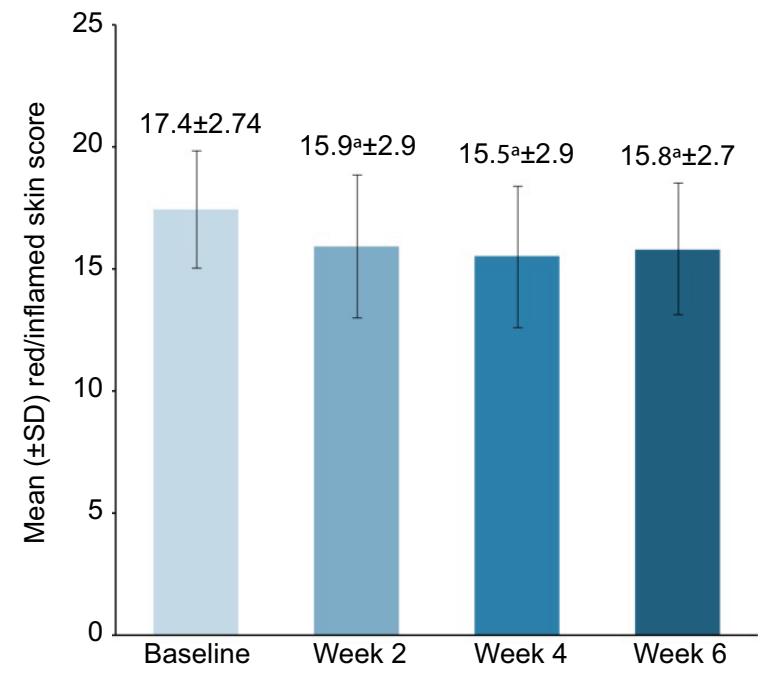

began using the anti-acne 3-step, twice-daily skincare regimen $(P<0.05$; Figure 2$)$. The mean percentage changes (improvement) from baseline at weeks 2,4 , and 6 were $8.6 \%, 10.9 \%$, and $9.2 \%$, respectively (Figure 2). Red/inflamed skin improved in $70 \%(\mathrm{n}=21)$ of subjects by week 2 increasing to $73 \%(\mathrm{n}=22)$ and $80 \%(n=24)$ of subjects by weeks 4 and 6 , respectively.

There were statistically significant decreases (improvements) for open and closed comedones and papules at all time points and for nodules at week 6 compared to their respective baselines $(P<0.05$; Figure 3$)$. The percentage of these improvements ranged from $23.5 \%$ to $54.5 \%$ for open

\section{B}

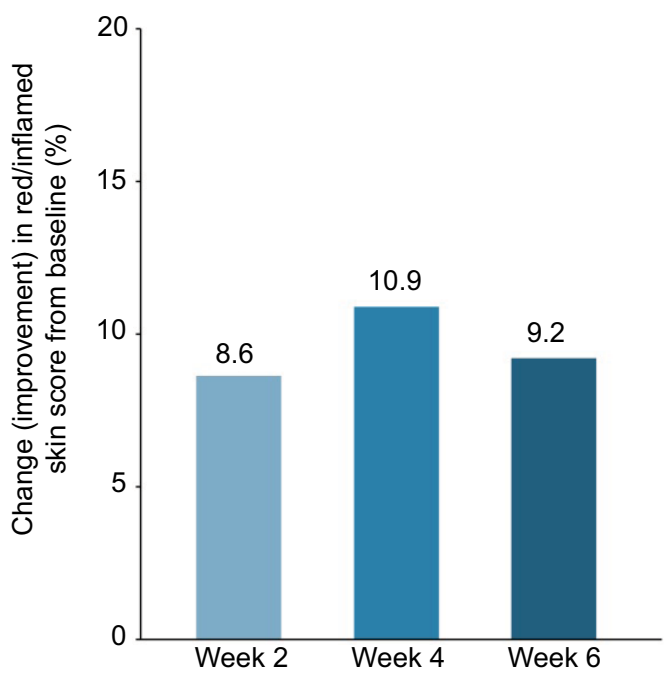

Figure 2 Improvement in (A) mean red/inflamed skin scores and (B) percentage change from baseline of red/inflamed skin in subjects who used twice-daily, 3-step anti-acne skincare regimen.

Note: ${ }^{a} P<0.05$ versus baseline. Visia-CR ${ }^{\circledR} /$ mage-Pro ${ }^{\circledR}$ analysis.

Abbreviation: SD, standard deviation.
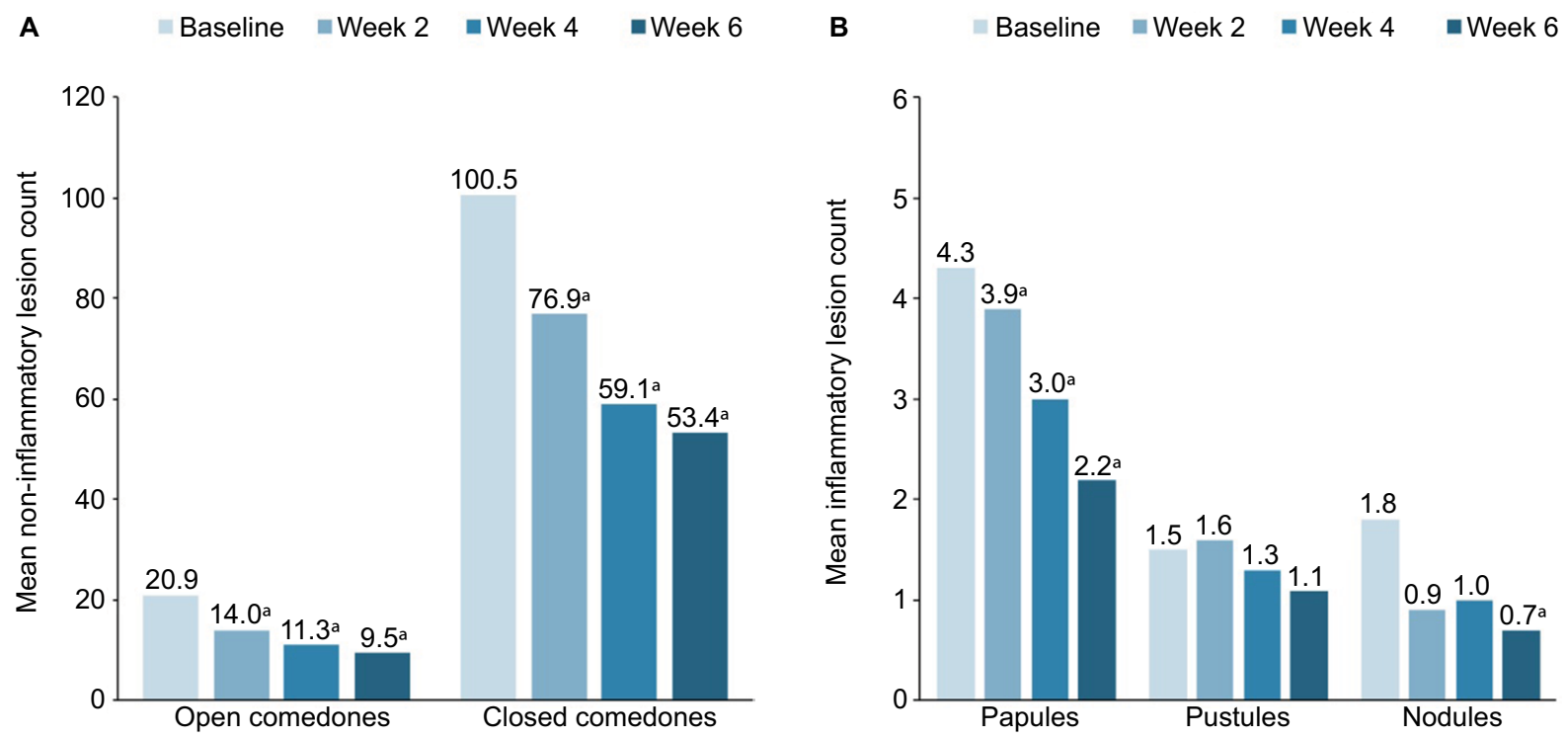

Figure 3 Improvement in mean (A) noninflammatory and (B) inflammatory lesion counts in subjects who used a twice-daily, 3-step anti-acne lesion count in subjects who used twice-daily, 3-step anti-acne skincare regimen.

Note: ${ }^{a}<0.05$ versus baseline value of the same category. Visia-CR ${ }^{\circledR} /$ mage-Pro $^{\circledR}$ analysis. 


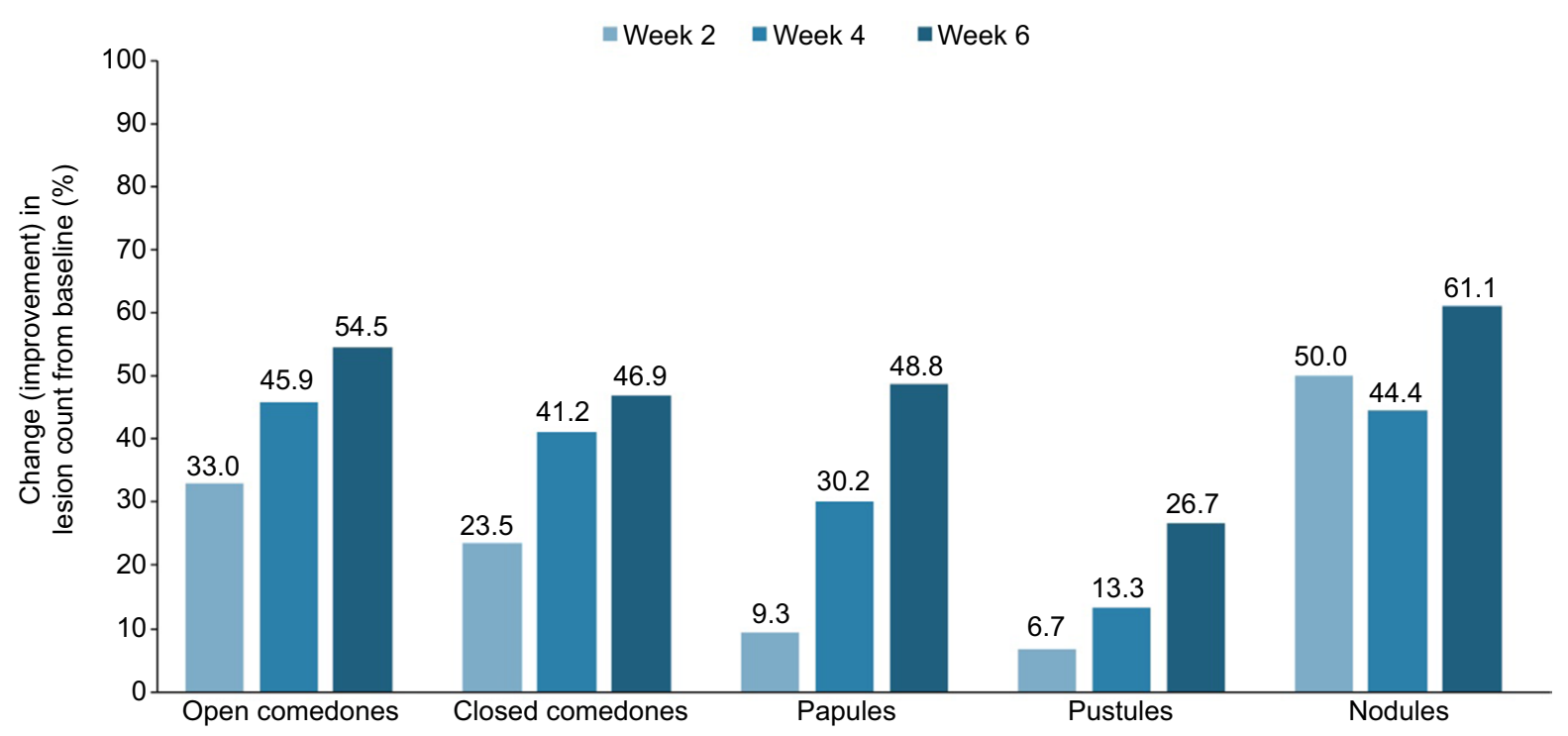

Figure 4 Improvement in percentage change in lesion count from baseline in subjects who used twice-daily, 3-step anti-acne skincare regimen. Note: Visia-CR ${ }^{\circledast} /$ mage-Pro ${ }^{\circledR}$ analysis.

and closed comedones and from $9.3 \%$ to $48.8 \%$ for papules (Figure 4). Although improvement of pustules at weeks 2, 4 , and 6 was not significant, they improved by $6.7 \%, 13.3 \%$, and $26.7 \%$ from baseline, respectively. Nodules were improved by $50 \%$ at week 2 and $44.4 \%$ at week 4 (Figure 4 ). Compared with baseline, $57 \%-100 \%$ of subjects showed improvement in open and closed comedones and papules after 6 weeks of the anti-acne skincare regimen. Up to $27 \%$ and $37 \%$ also showed improvement in pustules and nodules, respectively, during the study.

No skin irritation was observed in any subject at any study time point. No irritation, discomfort, or other safety concerns were noted by the investigator, reported directly from the subjects, or recorded in subject diaries.

\section{Discussion}

The objective of this trial was to determine the effectiveness of a twice-daily, OTC 3-step anti-acne skincare regimen in treating acne and reducing the appearance of red/inflamed facial skin. The findings from this study showed that after 6 weeks of consistent use, this twice-daily regimen demonstrated statistically significant efficacy in reducing acne grading and lesion counts, while improving subjects' overall appearance through reduction of red and inflamed skin.

Full benefits of all the parameters measured were seen as early as 2 weeks, and additional benefits were observed with continued use at 4 and 6 weeks. The finding of no significant improvements in pustules and nodules was most likely due to their relatively low mean scores at baseline, making changes harder to detect within a relatively limited number of subjects.
Although OTC acne regimens may cause local adverse effects, such as irritation and dryness, the 3-step skincare regimen in this clinical trial was well tolerated, with no skin irritation or adverse effects reported. Additional studies are needed to determine any side effects associated with long-term use.

When used together, the three steps of this skincare regimen target the four aspects of acne pathophysiology (increased sebum production, follicular hyperkeratinization, bacterial colonization, and inflammation). The beneficial ingredients in the tested regimen include sulfur 3\%, witch hazel, and benzoyl peroxide $5 \%$.

Sulfur, used in the cleanser, is a keratolytic agent, causing mild exfoliation. ${ }^{9}$ In addition, use of a synthetic detergentbased (syndets) cleanser can reduce the potential for cutaneous irritation that is sometimes associated with topical acne treatments and has been hypothesized to maintain the natural $\mathrm{pH}$ of the skin (5.3-5.9), thereby preventing the higher $\mathrm{pH}$ conditions that favor microbial growth. ${ }^{9}$ (Pure soaps can increase the skin's $\mathrm{pH}$ for 4-8 hours after use, creating favorable conditions for the proliferation of Propionibacterium acnes. $^{9}$ ) The toner's primary ingredient, witch hazel, is a botanical anti-inflammatory and a natural astringent that removes excess oil from the skin's surface. ${ }^{15}$

Benzoyl peroxide, used in the acne treatment, is recommended as a first-line acne therapy by the American Academy of Dermatology and has been well studied for its antibacterial properties against $P$. acnes. ${ }^{7,9,10}$ Previous studies have noted benzoyl peroxide's rapid onset of action, lack of associated bacterial resistance, and significant decreases in inflamed lesions observed in as soon as 5 days. ${ }^{16-18}$ 
Limitations of this study include the relatively small number of subjects and the short, 6-week duration, making it difficult to detect small changes and potential differences that might be seen in a larger population with continued, longterm use. Also, this study was not blinded and did not have a placebo-controlled arm for determining whether results could be attributed to the vehicles used in this multistep skincare regimen. However, previous research has established that use of a medicated anti-acne skincare routine is more effective in lessening acne severity compared with placebo. ${ }^{19}$ Finally, this study includes both adolescents and adults, which may not allow for possible age group physiological variations that could affect skin characteristics. ${ }^{20}$

Because of the variability in acne grading systems and observer bias from study to study, the use of digital imaging systems such as Visia-CR ${ }^{\circledR}$ and technologically advanced software in products, such as Image-Pro ${ }^{\circledR}$, is gaining industry acceptance. ${ }^{10,12,13}$

\section{Conclusion}

The American Academy of Dermatology recognizes the physical and psychological impact of acne and the beneficial impact that early, effective treatment has on the longterm emotional outlook of patients. ${ }^{10}$ This clinical study demonstrated the effectiveness of an OTC 3-step, anti-acne skincare regimen in significantly improving acne and the overall appearance of skin in the majority of subjects with mild-to-moderate acne. Given the high incidence of acne and individuals' propensity for self-treatment, the recommendation of medicated OTC products specifically targeting acne's complex pathophysiologic process is a worthwhile first-line approach for people with limited disease.

\section{Acknowledgments}

This study was funded by Rodan + Fields. Medical writing support was provided by Stephanie Eide and was funded by Rodan + Fields.

\section{Disclosure}

All authors are employees of Rodan + Fields. The authors report no other conflicts of interest in this work.

\section{References}

1. Gieler U, Niemeier V, Kupfer J. Acne and quality of life. J Eur Acad Dermatol Venereol. 2015;29(Suppl 4):12-14.

2. Dreno B, Poli F. Epidemiology of acne. Dermatology. 2003;206(1):7-10.

3. Titus S, Hodge J. Diagnosis and treatment of acne. Am Fam Physician. 2012;86(8):734-740.

4. Timms R. Moderate acne as a potential barrier to social relationships: myth or reality? Psychol Heal Med. 2013;18(3):310-320.

5. Lee IS, Lee AR, Lee H, et al. Psychological distress and attentional bias toward acne lesions in patients with acne. Psychol Health Med. 2014;19(6):680-686.

6. Mallon E, Newton JN, Klassen A, Stewart-Brown SL, Ryan TJ, Finlay AY. The quality of life in acne: a comparison with general medical conditions using generic questionnaires. $\mathrm{Br} J$ Dermatol. 1999;140(4):672-676.

7. Kraft J, Freiman A. Management of acne. Can Med Assoc J. 2011; 183(7):E430-E435.

8. Cheng CE, Irwin B, Mauriello D, Liang L, Pappert A, Kimball AB. Self-reported acne severity, treatment, and belief patterns across multiple racial and ethnic groups in adolescent students. Pediatr Dermatol. 2010;27(5):446-452.

9. Decker A, Graber EM. Over-the-counter acne treatments: a review. J Clin Aesthet Dermatol. 2012;5(5):32-40.

10. Zaenglein AL, Pathy AL, Schlosser BJ, et al. Guidelines of care for the management of acne vulgaris. J Am Acad Dermatol. 2016;74(5): 945-973.

11. Cook CH, Centner RL, Michaels SE. An acne grading method using photographic standards. Arch Dermatol. 1979;115(5):571-575.

12. Xu DT, Yan JN, Cui Y, Liu W. Quantifying facial skin erythema more precisely by analyzing colour channels of the VISIA red images. J Cosmet Laser Ther. 2016;18(5):296-300.

13. Patwardhan SV, Kaczvinsky JR, Joa JF, Canfield D. Auto-classification of acne lesions using multimodal imaging. J Drugs Dermatol. 2013; 12(7):746-756.

14. Chajra H, Redziniak G, Auriol D, Schweikert K, Lefevre F. Trihydroxybenzoic acid glucoside as a global skin color modulator and photo-protectant. Clin Cosmet Investig Dermatol. 2015;8: 579-589.

15. Chularojanamontri L, Tuchinda P, Kulthanan K, Pongparit K. Moisturizers for acne: what are their constituents? J Clin Aesthet Dermatol. 2014;7(5):36-44.

16. Schutte H, Cunliffe WJ, Forster RA. The short-term effects of benzoyl peroxide lotion on the resolution of inflamed acne lesions. Br J Dermatol. 1982;106(1):91-94.

17. Mills OH, Kilgman AM, Pochi P, Comite H. Comparing $2.5 \%, 5 \%$ and $10 \%$ benzoyl peroxide on inflammatory acne vulgaris. Int J Dermatol. 1986;25(10):664-667.

18. Chalker DK, Shalita A, Smith GJ, Swann, RW. A double-blind study of the effectiveness of a $3 \%$ erythromycin and $5 \%$ benzoyl peroxide combination in the treatment of acne vulgaris. J Am Acad Dermatol. 1983;9(6):933-936.

19. Zander E, Weisman S. Treatment of acne vulgaris with salicylic acid pads. Clin Ther. 1992;14(2):247-253.

20. McCarty M. Evaluation and management of refractory acne vulgaris in adolescent and adult men. Dermatol Clin. 2016;34(2): 203-206. 


\section{Supplementary material \\ SI: Subject instructions on the use of test materials}

Instructions: Dates and times of use must be included in this diary:

1. Record each time (AM and PM) that the test regimen was used.

2. Do not use any acne products other than the ones that are provided to you during the entire study.

3. Record any comments you may have (if any) in the space provided below.

\section{Directions:}

Regimen:

Every morning: Use Steps 1, 2, and 3 following directions. As needed, use the optional provided moisturizer after Step 3, if dryness or redness occurs. If you use a sunscreen, please use the one provided after Step 3 or moisturizer.

Every evening: Use Steps 1, 2, and 3 following directions. As needed, use the optional provided moisturizer after Step 3, if dryness or redness occurs.

\section{Product directions}

Step 1: Cleanser - Wet skin with lukewarm water. Using fingertips, gently and evenly massage a quarter-sized amount onto the entire face for 30-60 seconds. Avoid eye area and contact with eyes. Rinse thoroughly; pat dry.

Step 2: Toner - Apply evenly to the entire face with a gauze pad, avoiding the eye area. Do not rinse off. Allow to dry.

Step 3: Treatment: Dispense 1-2 pumps from each side of the treatment container and evenly apply over the entire face, avoiding the eye area. Do not rinse off. Allow to dry.

This product contains two separate formulas, a cream and a gel, that are dispensed individually from each side of the package. When using this product, make sure both the cream and the gel are dispensed equally in a 1:1 ratio (equal amounts). On the first use, you may need to pump a few times before the product dispenses properly.

Optional moisturizer: Gently pat on a small amount to dry or red areas. Use only as needed, if dryness or redness occurs.

Optional sunscreen: If you use a sunscreen, please use the one provided after Step 3 or moisturizer.

Note: Please record in your diary if/when you use the moisturizer and/or sunscreen.

Clinical, Cosmetic and Investigational Dermatology

\section{Publish your work in this journal}

Clinical, Cosmetic and Investigational Dermatology is an international, peer-reviewed, open access, online journal that focuses on the latest clinical and experimental research in all aspects of skin disease and cosmetic interventions. This journal is included on PubMed. The manuscript management system is completely online
Dovepress

and includes a very quick and fair peer-review system, which is all easy to use. Visit http://www.dovepress.com/testimonials.php to read real quotes from published authors 